\title{
The Mathematically Describable ILD Patterns
}

\author{
Balemir Uragun* \\ Monash University, Melbourne, Victoria, Australia. \\ * Corresponding author. Tel.: +90 531 2737550; email: uragun@hotmail.com \\ Manuscript submitted September 29, 2017; accepted November 3, 2017. \\ doi: 10.17706/ijbbb.2018.8.3.132-146
}

\begin{abstract}
This is a mathematical modelling study in computational science that recognized patterns used based on the biological data. The biological data were obtained through the number of clusters and the shape of each clustered means Interaural Level Differences (ILD) and were both analytically examined by the data classification study. Then the result of the classification study trained by the Artificial Neural Networks to build a master-template for a single array. Here, these typified single array patterns were exclusively tested for several curve fitted functions and the outcome was the probability density functions " $p d f$ " with the linear regression parameters. This initial evaluation confirms two Gaussian functions were both suitable models for the data sets, and then coefficients of these functions verified for a correlation to be validated. In conclusion, a parameterized first-order Gaussian function can be used as the mathematical model for all ILD patterns; the energy efficiency is also discussed.
\end{abstract}

Key words: Energy efficiency, Gaussian function, machine learning, mathematical modelling.

\section{Introduction}

This paper introduces a new approach to develop a mathematical modelling from clustered data. There have been many modelling applications in machine learning, and considering to derive an algorithm from biological data sets; for recently examples, using the taxonomic levels of microbiomes information that helps to predict the time of death of the human cadaver [1] or predicting relationship trouble from vocal patterns in couples' speech [2], or developing hearing devices [3]. The patterns used for this study also came from the biological data sets, in next subsection.

\subsection{Recognized Patterns: The Biological-data, ILD}

One of the sound localization cues is the ILD; these common shapes of ILD sensitivity functions as recognized patterns have been classed in four "general" forms [4]. These four classical patterns) are namely Insensitive, Peaked, Sigmoidal-IE, and Sigmoidal-EI, and show similarity across four important auditory nuclei. However, in a previous ILD data classification study showed that the "seven" number and "similar" types of generic ILD function patterns methodologically verified. This following method is a non ad hoc basis and mapped well on to the electrophysiologically determined ILD sensitivity functions [5]. In this analytical work, the data were collected from the central part of the inferior colliculus (ICc), (it is the certain part of the rats' brain used as an experiment animal), and the ILD sensitive neurons have been observed and the formation of ILD patterns occurred in the auditory nuclei. These recognized patterns in the ICc also show similar patterns in other mammals such as in cats, bats or in rats [6]-[8].

The justification of ICc selection for the data; several auditory nuclei contain cells that code binaural cues 
and therefore are likely to be involved in sound localization. The data collected for "ILD patterns" have come from the center of IC; the IC has been focused upon because (a) it is an obligatory auditory nucleus both in addition to general and in respect to sound localization in particular, (b) it is the site at which binaural cues converge in at least some species [9], (c) it is easy to collect electrophysiological data on ILD coding and such coding here is very similar to that seen within the auditory cortex, and (d) although ILD coding is present in sub-colliculi structures, there is good evidence that it is generated de novo within the IC, suggesting that this is an important structure in ILD-based sound localization.

\subsection{Using Curve Fitting Techniques}

A literature search for the data curve fitting application was carried out and with the limited results found in the auditory related studies. For example, Gaussian and Sigmoidal curve fitting functions were used to compare the relationship between discharge rate and azimuthal sound location and the goodness of fit also examined by the correlation of these curve fitting function coefficients [10]. Although the Groh's method was similar to this methodology, the motivation of the study was not developing the mathematical model. Few other curve fitting applications are; in the auditory filter bank design that used a least-squares criterion to fit a $7^{\text {th }}$ order polynomial curve fit to data [11], and another filter design application which was used in tuning the curves of an auditory nerve fiber utilizing the filter of a $9^{\text {th }}$ order nominator and $9^{\text {th }}$ order denominator fit to each measured data [12]. A similar application was in the evoked potential, using an exponential curve fit by [13]. Another, exponential curve fit case was previously used by Ponton, but was replaced with a least-square single time-constant nonlinear curve fit for the brain-stems auditory response signals data [14]. The other model looks at how, the nonlinear curve fits into the auditory averaged electroencephalic responses data [15], or how a waveform from the motion of the swim-bladders of fish in the Peripheral auditory systems mechanics fits; which was using a least-squares technique [16].

The most related curve fitting method to this technique was compared: In a study of intensity coding for frogs it was shown that calculating dynamic range based on the curve fitted function of a monotonic rate-intensity function [17]. Eggermont utilized a sigmoidal function to fit the data, using different variations of standard deviation. This approach was also applied to the parameters of the $p d f$ models. This curve fitting technique was previously proposed in the use of automatic pattern recognition for the Auditory Brainstem Response (ABR) waveform, [18]. The number of curve fitted functions examined to select the suitable function to be modeled for ABR. The result was the parameter of this mathematical modelling of ABR pattern, and suggested to utilize as an automatic health diagnostic tool.

\section{Method}

A two-step procedure was applied for Neural Network trained clustered data "seven group of ILD patterns," using the curve fitting toolbox of MATLAB. In this manuscript, the result from the Neural Network trained clustered data were used as a master-template, instead of averaging clustered data-points as were in [18] with reasons detailed in the [19]. The first step is to find the suitable curve fitting functions if it is appropriate for the master-template then second step is to assess the first step's result for filtering in some of the curve fitting functions, namely $1^{\text {st }}$ and $2^{\text {nd }}$ order Gaussian functions. This curve fitting assessment is simply carried out a group of statistical data-analysis including the linear regression for varying the coefficients of the curve fitted functions. The results from the goodness of fit, confidence limits, and linearity tests; they all were used to verify the suitability of the curve fitted function. The variations of the Gaussian coefficients were examined to find any systematic differences or linear relations (correlations) between each of clustered data. This examination was necessary to build a mathematical model for all ILD patterns where towards a generalization work was based on the relationship of these varying coefficients.

Using $p d f$ on the estimation technique had been previously established, [20]. Here, the result of the first 
step needed to find out if the $p d f$ (the normal distribution) [21]) with two parameters, namely " $\mu$ " mean and " $\sigma$ " standard deviation (or $\sigma^{2}$ as a variance) were both capable of producing normalized ILD patterns. The systematically variations of these two parameter values of $p d f$ produced 432 different types of plots and 252 of them plots was visually examined for the suitable in seven "normalized" ILD patterns, Appendix.
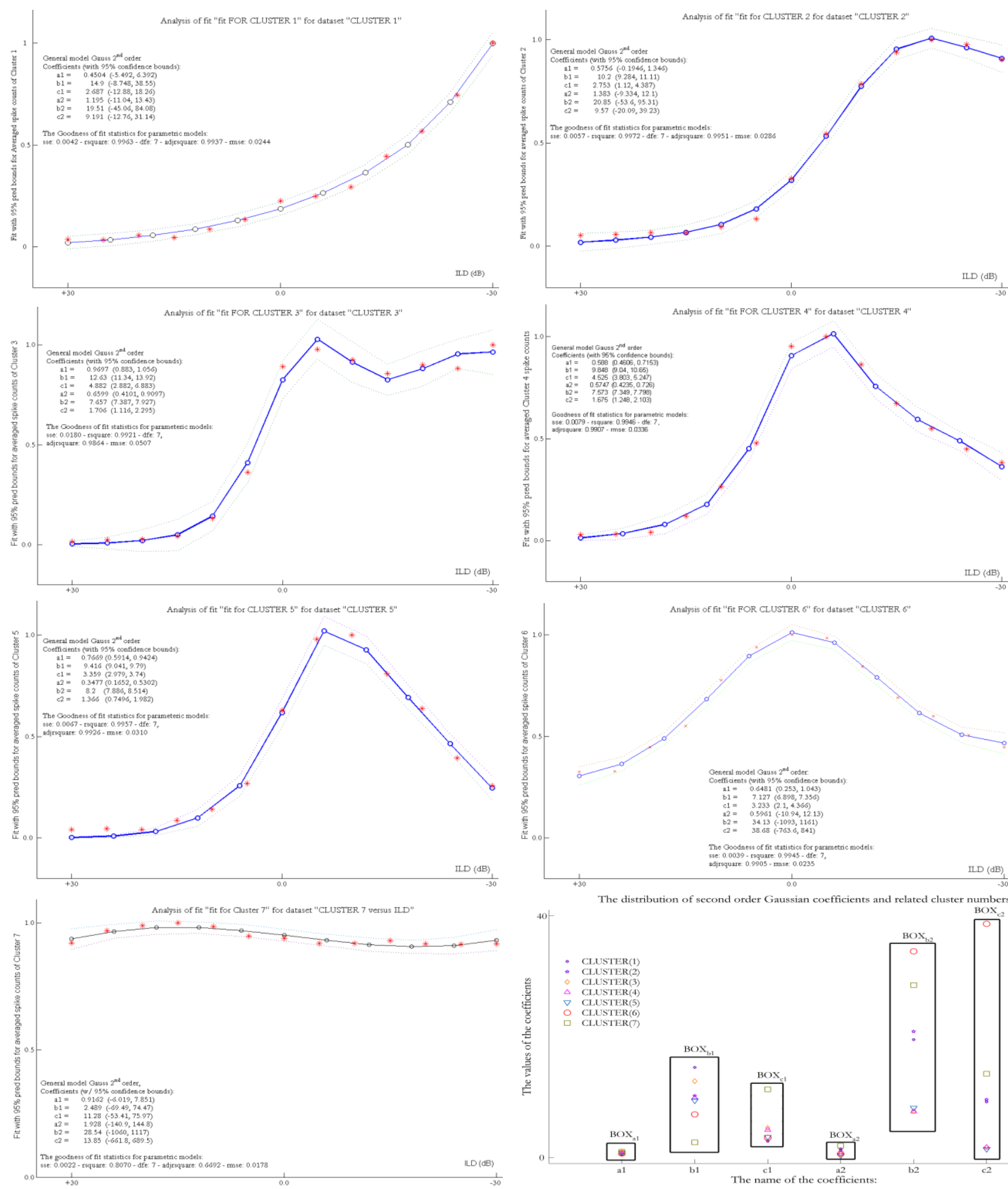

Fig. 1. ANN trained Cluster 1 through 7 data (asterisk) points and fitted second-order Gaussian model data (solid line) within 95\% confidence limits were organized by seven windows; more details are in the Appendix. 
Those two sets of seven parameter values were tested for their linearity, and the result of the linearity assessment helped to select the suitable curve fitting function from the $1^{\text {st }}$ and $2^{\text {nd }}$ order Gaussian functions. The next step was to resolve which Gaussian functions would be a more appropriate choice for all ILD patterns that was also based on the linearity test for their seven sets of coefficients.

Table 1. The Values of Six Coefficients from the 2nd Order Gaussian Curve Fit

\begin{tabular}{ccccccc} 
Cluster & \multicolumn{5}{c}{ The coefficients of a 2nd $^{\text {nd }}$ order Gaussian model } \\
No: & $\mathrm{a}_{1}$ & $\mathrm{~b}_{1}$ & $\mathrm{c}_{1}$ & $\mathrm{a} 2$ & $\mathrm{~b}_{2}$ & $\mathrm{c}_{2}$ \\
\hline$\# 1$ & 0.4504 & 14.899 & 2.6866 & 1.1955 & 19.509 & .1909 \\
$\# 2$ & 0.5755 & 10.198 & 2.7534 & 1.3832 & 20.855 & 7.5695 \\
$\# 3$ & 0.9696 & 12.630 & 4.8823 & 0.6599 & 7.6572 & 1.7055 \\
$\# 4$ & 0.5879 & 9.8477 & 4.5252 & 0.5747 & 7.5732 & 1.6753 \\
$\# 5$ & 0.7669 & 9.4160 & 3.3590 & 0.3477 & 8.2000 & 1.3660 \\
$\# 6$ & 0.6481 & 7.1268 & 3.2330 & 0.5961 & 34.132 & 38.681 \\
$\# 7$ & 0.9161 & 2.4890 & 11.280 & 1.9281 & 28.541 & 13.853 \\
\hline
\end{tabular}

\subsection{Mathematical Representation of the Group of Data Using Curve Fitting}

The curve fitted for each of the seven patterns of ILD functions (master-template) were initially tested by the curve fitting toolbox to find the suitable Gaussian function. The $2^{\text {nd }}$ order Gaussian function with six-coefficients was presented (1), and the fit options for the Gaussian models parameters and goodness of fit statistics were also explained in detail, Appendix. The $2^{\text {nd }}$ order Gaussian functions used in the model fitted all seven data cluster sets. The coefficients $\left(a_{1}, a_{2}, b_{1}, b_{2}, c_{1}\right.$, and $\left.c_{2}\right)$ varied for each seven ILD patterns.

$$
f(x)=a_{1} \mathrm{e}^{-\left(\frac{x-b_{1}}{c_{1}}\right)^{2}}+a_{2} \mathrm{e}^{-\left(\frac{x-b_{2}}{c_{2}}\right)^{2}}
$$

The coefficients of the $2^{\text {nd }}$ order Gaussian functions vary by the fit to each of the master-template, and the values of these coefficients were tabulated in Table I. Each set of coefficients was also included within the inset of the figures (Fig. 1). The default values of the parameters for the curve-fitted function were tabulated in the Appendix. The analysis of fitted curves for the master-template resulted in seven sets of coefficients where these values are presented in Table I and insets of the figures (Fig. 1). Two groups of seven curve-fitted coefficients were also depicted in six boxes (Fig. 1) and their linearity examination in Fig. 2. The linearity examination was tested for each group of six coefficients, which were obtained from the curve fitted functions. The $1^{\text {st }}$ order polynomial model Least Squares Fit Line (LSFL) was applied to these groups, one-by-one. For example, the first group of seven " $a_{1}$ " coefficients was presented in "BOXa1" in Fig. 1, as well as the linearity test of seven " $a_{1}$ " coefficients in the first window of Fig. 2 . In the end, the resulting linearity tests showed that six groups of seven coefficients all varied in linear variation according to the $1^{\text {st }}$ order polynomial model LSFL, (Table II). The results from the $1^{\text {st }}$ order polynomial model showed seven coefficients and so their curve fitted data were linearly related to each other. Each " $p_{1}$ " and " $p_{2}$ " values for fit line were termed for the coefficients.

Table 2. First-Order Polynomial $\left(p_{1} \cdot \mathrm{x}+p_{2}\right)$ Model Used for Testing the Linearity of Six Coefficients

\begin{tabular}{ccccccc} 
& \multicolumn{5}{c}{ COEF F IC IEN T S } \\
\cline { 2 - 7 } Polynomial & $\mathrm{a}_{1}$ & $\mathrm{~b}_{1}$ & $\mathrm{c}_{1}$ & $\mathrm{a}_{2}$ & $\mathrm{~b}_{2}$ & $\mathrm{C}_{2}$ \\
$p_{1}$ & 0.0478 & -1.664 & 0.9006 & 0.0111 & 1.9350 & 2.5670 \\
$p_{2}$ & 0.5107 & 16.170 & 1.0720 & 0.9105 & 10.320 & 0.5961 \\
\hline
\end{tabular}

The coefficients from the $2^{\text {nd }}$ order Gaussian curve fit were all positive numbers that varied between +0.34 and +38.68 . These values are also presented in a group of six boxes (Fig. 1), with their linearity 
examination in Fig. 2. On the left-hand side of Fig. 2, three windows showed the coefficients of " $a_{1}$ ", " $b_{1}$ ", and " $C_{1}$ ", which were from the first part of (1). On the right-hand side of the same figure (Fig. 2) three windows showed the coefficients of " $a_{2}$ ", " $b_{2}$ ", and " $c_{2}$ ", which appeared as the second part of the same equation, (1). The LSFL is applied for each group of the coefficients as a linearity test, Fig. 2 . The linearity test of the coefficients was separately examined (i.e.; seven " $a_{1}$ " from seven ILD data clusters, seven " $b_{1}$ " from seven ILD data clusters, etc.) six coefficients were tested by the "LSFL" method. All 42 coefficients (six coefficients for each of the seven ILD data clusters) were tabulated in Table I. Each variation was fitted for each set of coefficient by the $1^{\text {st }}$ order polynomial model; their coefficients (" $p_{1}$ " and " $p_{2}$ ") are both expressed in Table II. The statistical results are expressed in terms of goodness of fit for a LSFL versus each coefficient from the $2^{\text {nd }}$ order Gaussian model depicted in Fig. 2. The closer the values of SSE and RMSE are to zero the better the fit, in contrast to a higher value for the $\mathrm{R}^{2}$ and Adj. $\mathrm{R}^{2}$ approaching one being a better fit (Appendix) for the $1^{\text {st }}$ order polynomial model of the coefficients $\left(a_{1}, b_{1}, c_{1}, a_{2}, b_{2}\right.$, and $\left.c_{2}\right)$, Table 3 .
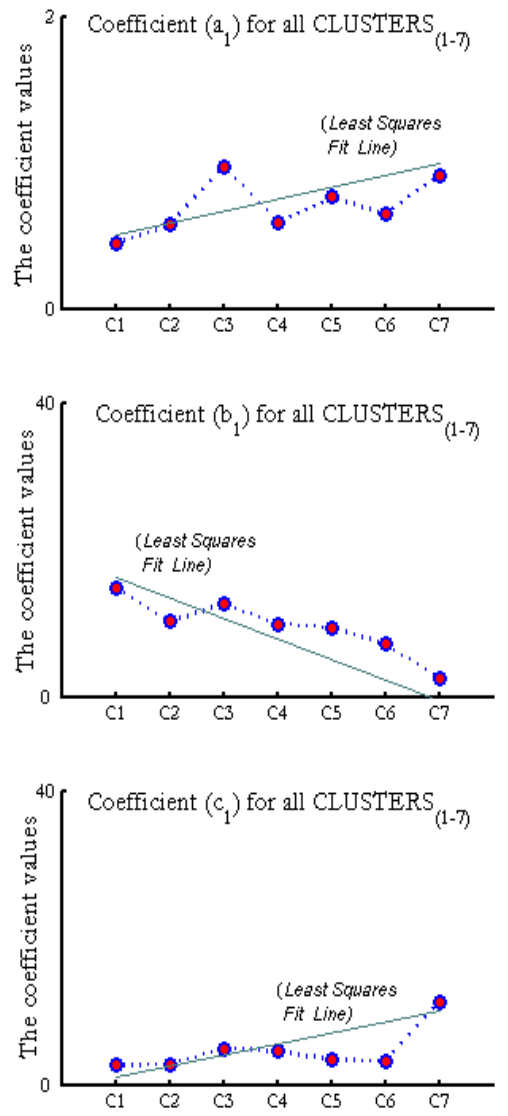

The variation of six coefficients for seven clusters

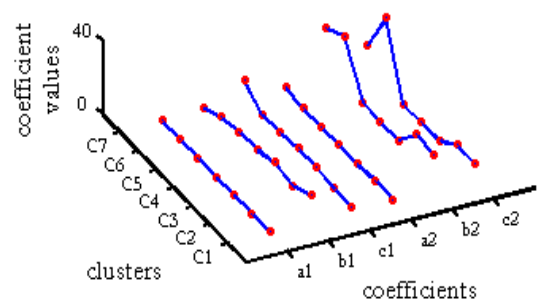

The coeff. of $2^{\text {nd }}$ order Gaussian function

$f_{(x)}=a_{1} \cdot e^{-\delta_{1}}+a_{2} \cdot e^{-\delta_{2}}$
$\delta_{1}=\left[\left(x-b_{1}\right) / c_{1}\right]^{2}$
$\delta_{2}=\left[\left(x-b_{2}\right) / c_{2}\right]^{2}$
$x \in \Re$
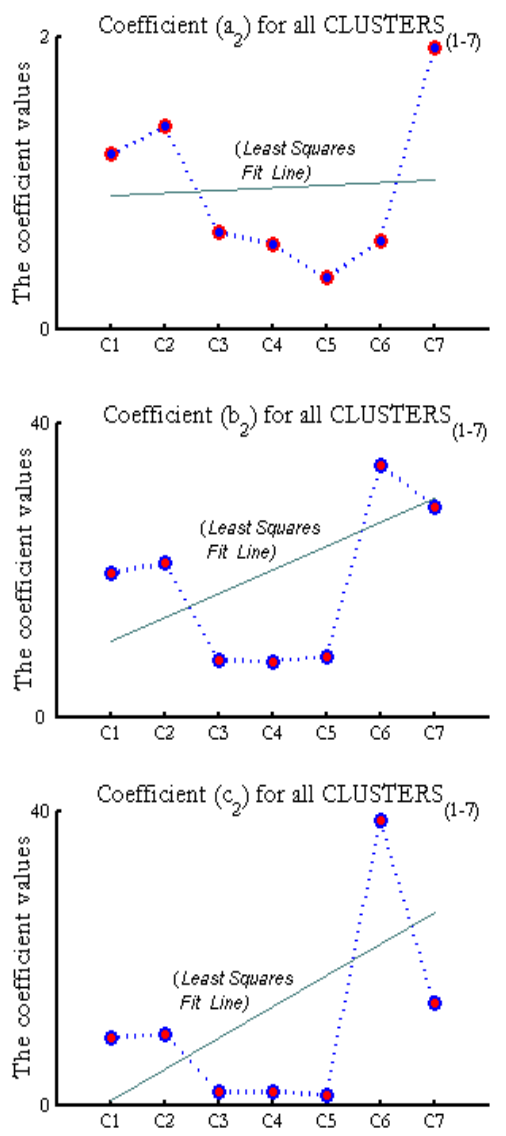

Fig. 2. The coefficients of a 2 nd order Gaussian curve fit are presented for each of the 7 ILD patterns. These fitted values of the 6 coefficients were individually grouped for the comparison; more details are in the Appendix.

\section{Results}

In summary, the ILD patterns were reviewed as clustered data; for ongoing high frequency sounds, the major cue is the difference in intensities produced at the two ears as a sound moves around the head. In mammals, it appears that this cue is first functionally coded by neurons in the auditory midbrain and occurs through a variety of interactions between excitatory and inhibitory inputs ultimately emanating from the two ears in mammals as one of the important sound localization cues. In a previous data-classification work 
[5] using ILD data, which was extracted from the extracellular recordings that were collected from a rat's IC.

A brief description of a previous data classification study was explained that averages were first verified from all ILD patterns in each of the seven clustered data to identify the prototypical ILD pattern in that cluster. Then, statistical data analysis methods were applied to differentiate between the ILD patterns. The result disclosed seven different prototypical ILD patterns, found from the three broad categories of ILD patterns, namely peak, sigmoidal (two types "EI" \& "IE" of them) and insensitive ILD patterns. Nearly 85\% of the electrophysiological data were of the peak and sigmoidal type of ILD patterns. These analyses were completely congruent with the Cluster Analysis and the seven ILD pattern types from statistical analyses corresponded very well with the seven ILD pattern types determined by a previous Cluster Analysis [5].

Table 3. The Statistical Analysis of the LSFL for 2nd Order Gaussian Model

\begin{tabular}{|c|c|c|c|c|c|c|}
\hline \multirow{2}{*}{$\begin{array}{l}\text { Yoodness } \\
\text { of fit }\end{array}$} & \multicolumn{4}{|c|}{$\begin{array}{r}\text { C O E F F I C I E N T S } \\
\text { Gaussian) }\end{array}$} & \multicolumn{2}{|c|}{$\left(2^{\text {nd }}\right.$ order } \\
\hline & $\mathrm{a}_{1}$ & $\mathrm{~b}_{1}$ & $\mathrm{c}_{1}$ & $\mathrm{a}_{2}$ & $b_{2}$ & $\mathrm{C}_{2}$ \\
\hline SSE: & 0.1528 & 16.840 & 32.440 & 1.9140 & 588.60 & 861.20 \\
\hline $\mathrm{R}^{2}:$ & 0.2955 & 0.8215 & 0.4118 & 0.0018 & 0.1513 & 0.1764 \\
\hline Adjuste & $2: 0.1546$ & 0.7858 & 0.2941 & -0.197 & -0.018 & 0.0116 \\
\hline RMSE: & 0.1748 & 1.8350 & 2.5470 & 0.6187 & 10.850 & 13.120 \\
\hline
\end{tabular}

Table 4. The Statistical Analysis of the LSFL for PDF

$$
1 \text { st ORDER POLYNOMIAL }\left(p_{1} \cdot x+p_{2}\right)
$$

The model used for Least Square Fit Line

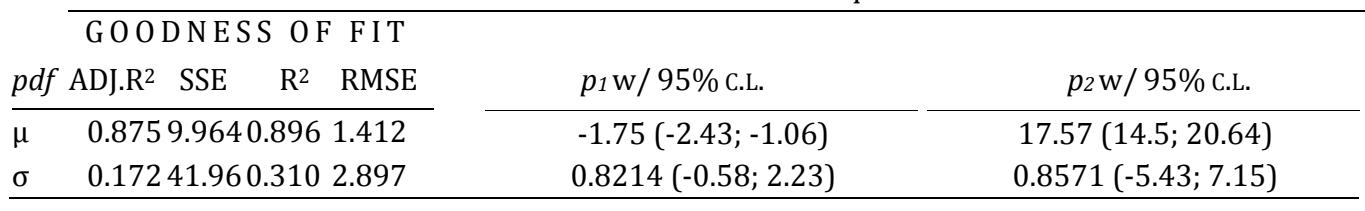

Curve fitting has been used for many applications where it may be categorized as a parametric and non-parametric data fitting procedure. Due to the results of the many $p d f$ observations, a Gaussian type of parametric fit was suitable for seven clusters of ILD data; this observation was also verified by the linear variations of two parameters, " $\mu$ " and " $\sigma$ ". Then $2^{\text {nd }}$ order Gaussian function (1) is applied to the master-template. The results of six coefficients obtained from a $2^{\text {nd }}$ order Gaussian curve fit model presented two issues; (i) the linearity examination of the data and (ii) a $1^{\text {st }}$ order Gaussian fit was sufficient for data use. The $2^{\text {nd }}$ order Gaussian fit was (too generic) statistically less descriptive and had difficulty interpreting the ILD data model. Therefore, a $p d f$ for ILD data patterns was revised for the $1^{\text {st }}$ order Gaussian model that uses two statistical parameters (standard deviation and mean) instead of using just two-set of three parameters $\left(\mathrm{a}_{1,2}, \mathrm{~b}_{1,2}\right.$ and $\left.\mathrm{c}_{1,2}\right)$.

1) The spike counts of each ILD data points were resulted from the Neural Network trained seven clustered data (e.g., the "Cluster 3" was composed by 21 similar types of ILD patterns, Appendix - Fig. 8; the ILD patterns incidentally showed similarities within the results of averaged ILD data-points).

2) Gaussian curve fitted functions were suitable for all seven ILD patterns, within other curve fitted functions (i.e. exponential, hyperbolic, sum of exponentials, and etc.).

3) The coefficients of curve fitted (a) $2^{\text {nd }}$ order Gaussian function, (b) $1^{\text {st }}$ order Gaussian function, and (c) the variation of the $p d f$ parameters were all statistically examined; the goodness of the curve fit was within the $95 \%$ confidence bounds.

4) The statistically descriptive values for these coefficients were obtained with the curve fitted functions, and the values were compared to conclude the mathematical model for the ILD patterns.

\subsection{The Result of the $2^{\text {nd }}$ Order Gaussian Curve Fitting}


The seven ILD patterns were used as a master-template to find a suitable curve fit model for each group, using the curve fitting toolbox 1.1 of MATLAB (Appendix). There were two reasons to apply curve fitting to the seven clustered data: $(a)$ in order to examine the correlation among these seven data parameters, which are continuous variables, and (b) to convert the discrete data (i.e. ILD functions) into a continuous variable. The $2^{\text {nd }}$ order Gaussian function was initially nominated as a model for all seven clustered data within $95 \%$ of two (upper and lower) confidence limits, as shown in Fig. 1. The next stage, involved a search for linearity of the coefficients from the Gaussian model, these coefficients were observed in six different groups (Fig. 1) to show the distribution of coefficients. These coefficients, especially " $b_{2}$ " and " $c_{2}$ ", were sparsely distributed and appeared in " $B O X_{b 2}$ " and " $B O X_{c 2}$ " in comparison to coefficients " $b_{1}$ " and " $c_{1}$ " which appeared in " $B O X_{b 1}$ " and "BOX ${ }_{c 1}$ " (Fig. 1).

1) The result of the set of coefficients distribution is shown as a box representation (Fig. 1) and is examined by the coefficients of a $2^{\text {nd }}$ order Gaussian curve fit (Table I).

2) The result of the LSFLs in Fig. 2 shows linear fitting as a more suitable option for the " $a_{1}$ ", " $b_{1}$ ", and " $c_{1}$ " (first three set) coefficients than the (second three set) " $\mathrm{a}_{2}$ ", " $\mathrm{b}_{2}$ ", and " $\mathrm{c}_{2}$ " coefficients.

3) The result of the goodness of fit test parameters examined for the $1^{\text {st }}$ order polynomial "linear-fit" model in Table IV with, parameters are detailed in the Appendix.

The conclusion was induced from the obtained results of the three elements above; (i), (ii) and (iii) establishing that a $1^{\text {st }}$ order Gaussian function (2) is more suitable to use for ILD data model rather than using a $2^{\text {nd }}$ order Gaussian model. Although a $2^{\text {nd }}$ order Gaussian function curve fitting is adequate within the $95 \%$ confidence limits the linear variation of the first set of three coefficients (" $a_{1}$ ", " $b_{1}$ ", and " $c_{1}$ ") are better then the second set of three coefficients. In other words, a $1^{\text {st }}$ order Gaussian curve fit (2) or a similar type of normal distribution can be used as a model for clustered data representations. This result is also supported by one of the 10 different types of distributions that are "physiologically" mentioned among 200 interspike interval histograms [22].

$$
f(x)=a_{1} e^{-\left(\frac{x-b_{1}}{c_{1}}\right)^{2}}
$$

\subsection{The Results of the $p d f$, and $1^{\text {st }}$ Order Gaussian Curve Fit}

The $p d f$ of a normal distribution is the parameterized $1^{\text {st }}$ order Gaussian function (comparing the equations (3) to (2)) and because of this comparison a $p d f$ can be used as a model for ILD data. The application of $p d f$ distribution for the seven data clusters and its rational will be addressed. A $1^{\text {st }}$ order Gaussian function containing only three coefficients $\left(a_{1}, b_{1}\right.$, and $\left.c_{1}\right)(2)$. A $1^{\text {st }}$ order Gaussian curve fit is good enough to be used in ILD data, instead of using a $2^{\text {nd }}$ order Gaussian curve fit, expressed in (1).

The systematic variation of a normal $p d f$ parameters' value (" $\mu$ " and " $\sigma$ ") could help generate a different shape for a $1^{\text {st }}$ order Gaussian type function. The 13 data points, " $x$ " variables corresponded to an ILD function (a change from $+30 \mathrm{~dB}$ to $-30 \mathrm{~dB}$ with $5 \mathrm{~dB}$ decrements), and a number of spike counts which are represented by the output of a normal $p d f$ shown as a model. The ILD data clusters with their $p d f$ distribution models are both formed to be shown in Fig. 3. The linearity, changes of a models parameters ( $\mu$ " and " $\sigma$ ") are separately examined in the last two windows of Fig. 3. The values presented by " $\mu$ " express, means and " $\sigma$ " expresses the standard deviations linear variation, which is also supported by a LSFL. The $p d f$ of a normal distribution is a $1^{\text {st }}$ order Gaussian function. The $p d f$ depends on the " $x$ " variable with two other statistically significant parameters; " $\mu$ " and " $\sigma$ ".

$$
f(x \mid \mu, \sigma)=\frac{1}{\sqrt{2 \cdot \pi \cdot \sigma^{2}}} \mathrm{e}^{-\left(\frac{x-\mu}{\sqrt{2} \cdot \sigma}\right)^{2}}
$$



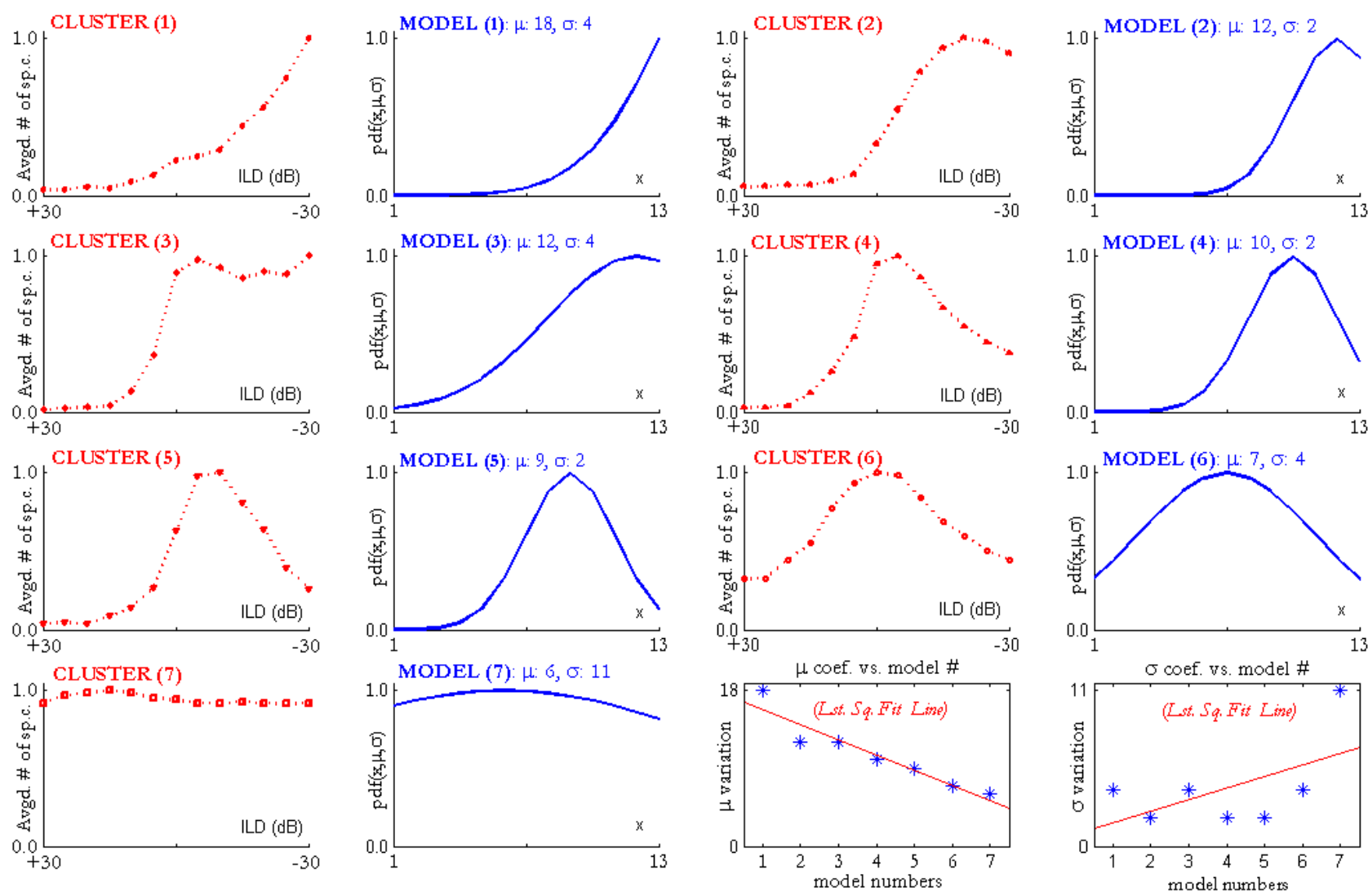

Fig. 3. The results of ILD clustered data are presented in dashed-lines. The normal $p d f$ distribution is used as a model, shown in solid-lines varied by the parameters of " $\mu$ " and " $\sigma$ " (these two parameter variations are both examined for the linearity by LSFL.

The conclusion between the ILD data clusters and a $p d f$ model for each cluster is correlated, and this correlation can be expressed as:

1) Seven ILD data clusters are constituted by a combination of excitation and inhibition effects of the neuronal inputs, which are shown in Appendix - Fig. 8. The inputs effect on Cluster 1 shows an ipsilateral inhibition and contralateral excitation. In next window, this excitation shows domination from contralateral to ipsilateral, and the ILD data cluster shapes turn into a sigmoidally shaped function (in the windows of Cluster 2 and Cluster 3). The balanced excitation and inhibition inputs for ipsilateral and contralateral are to produce a peaked type of formation (in the windows of Cluster 4 and Cluster 5). The plateau of a peaked type formation starts becoming flatter in Cluster 6 and turns out to be an insensitive type of ILD function in Cluster 7 this means that too strong of an excitation totally dominates both the ipsilateral and the contralateral. The transition from an Exponential through to a Sigmoidal then to a Peaked to an Insensitive type function can be expressed in a $1^{\text {st }}$ order Gaussian or $p d f$ type model with "statistically more informative" varying parameters.

2) These two " $\mu$ " and " $\sigma$ " parameters are varied to show seven models (from model 1 to model 7 ) that are compatible with seven ILD data clusters, in detail explained in the Appendix.

The LSFLs are used for the parameters ( $\mu$ " and " $\sigma$ ") of the $p d f$ distribution, and their goodness of fits are examined by the coefficient variations of these models, to see if they are linearly distributed. The $p_{1}$ and $p_{2}$ are within the $95 \%$ (C.L.) confidence limits. The variation of all " $\mu$ " values is shown to be more linear than that of the values produced from the " $\sigma$ ", this is also confirmed with the comparison of goodness of fit; "SSE"

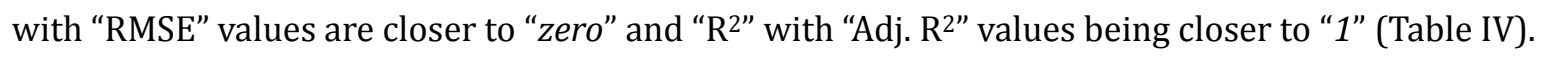

The LSFL is used to test the linearity of the statistical parameters ( $~ \mu$ " and " $\sigma$ ") within seven $p d f$ models. 
The linearity tests results show that seven standard deviations of $p d f$ models are not linearly distributed. On the other hand, seven " $\mu$ " values are linearly distributed for all seven $p d f$ models, Fig. 3. The linear variation of seven " $\mu$ " parameters is significantly informative for the $p d f$ type models variation. One of the "parameters" $p d f$ " $\sigma$ ", and the variation of the " $\sigma$ " values change exponentially, which does not validate the linearity test of the models. The non-linearity variations of " $\sigma$ " values in seven $p d f$ models are found to be inconclusive for this work. The reasons for an inconclusive result was; (i) the linearity test was not based on only " $\mu$ " parameters, and (ii) The " $\sigma$ " and " $\mu$ " parameters were both data dependent, meaning that these parameters (from a normal $p d f$ in (3)) could also be expressed as part of a cluster where the coefficients $\left(a_{1}\right.$, $b_{1}$, and $c_{1}$ ) of the $1^{\text {st }}$ order Gaussian function (2) are not data dependent. These coefficients are varied and only one out of the seven ILD data clusters were selected.

The resulting $1^{\text {st }}$ order Gaussian function is sufficient enough in serving as a seven clustered data model instead of using just normal $p d f$ distributions. The only disadvantage of a $1^{\text {st }}$ order Gaussian function is that it has three parameters when compared to a normal $p d f$ distribution with two parameters. However, the three coefficient values ( $a_{1}, b_{1}$, and $c_{1}$ ) of a $1^{\text {st }}$ order Gaussian function are all linear (Fig. 2), and this feature helps link the clustered-data variation. The Sigmoidal through to a Peaked to an Insensitive and others, which all together show seven ILD functions that can be modeled by a $1^{\text {st }}$ order Gaussian function. The variations of the three coefficients of a Gaussian function (increasing in " $a_{1}$ " and " $c_{1}$ " values while decreasing its " $b_{1}$ " value) can be concluded in defining the ILD functions with their transitions (from Sigmoidal through Peaked to Insensitive).

\section{Discussion}

In this section, the importance of mathematical modelling was briefed with a few examples. A recent trend in machine learning applications mentioned with the rapid progression of recognized patterns based algorithm development. This trend also refers "how the ILD patterns result that fits in the current understanding of mathematical modelling," here. Investigation the reasons behind the biological neural network's encoding mechanism shows the Gaussian type function, and the Gaussian type function hints the energy efficiency feature of the neuronal networks which is similar to the energy equilibrium of 128 years old Nernst's equation. The use of Gaussian functions has already been explored for wireless communication systems, where energy consumption increases significantly with distance.

\subsection{Development of a Mathematical Modelling from Recognized Patterns}

In nature, patterns show distinctive formations that can be recognized by their outlines (i.e. the shape of 2D or 3D objects). The configuration of such recognizable patterns is not only an emergent property of biological neural networks in the brain, but they can also be observed in nature. A good example of this is murmuration; a group of animals running away from a common predator displayed is a type of clustered pattern. This synchronized motion of animals is common among a flock of birds, school of fish, or a cloud of grasshoppers that can all be explained as herd behavior. Captivatingly, some of these crowd behavior models have already been expressed mathematically, as mentioned in [23]. The advantages of having the mathematical model of the system "in advance" can be beneficial (i.e. when a crop needs to be protected from the cloud of grasshoppers, or where the trade fishing becomes too competitive to catch fish).

Naturally there are a few good explanations, which form these types of patterns; the observation of patterns and its variations may carry cues for the system with its information. The information of patterns can therefore be a response characteristic of the system, where any fluctuation of the pattern is an indication of the changing steady state. For examples; birds fly in a "V" formation; this energy efficient behavior of a flock of birds is a pattern which helps conserve energy for each individual bird [24]. The flocks "total" energy efficient behavior can be mathematically deduced from the Bernoulli's equation with 
dimensional analysis [25]. Another example is the physiology of hearing organ and its sound localization capabilities are both closely linked to the statistics of naturally encountered binaural sounds [26]. Actually, the purpose of pattern recognition is to understand a way of representing an "event-actuated" perception statistically.

\subsection{Why Do We Need a Mathematical Model?}

The purpose of the math modelling can be underlined:

(i) To better understand the functionality of the system or to use as a knowledge enhancer,

(ii) To define a complex structure with a simplification using building blocks, and

(iii) To develop a mathematical expression which can be plausibly implemented within the real world.

Modelling is a powerful tool, and is capable in describing a system by mimicking its process; a system as simple as a mechanical or electrical process or even as complex as the human mind [27], human behavior [28], or a cluster of neuronal activity under stimulation as shown in this proceeding can be powerfully explained with the use of modelling. These specific activities can be observed as well as related to the patterns that can be recorded while under stimulus, for example in rats. These sequential patterns are the sign of activities occurring based on the stimulus.

Brain object recognition is a recognition task that is related to the neuronal firing patterns; this object recognition test is also confirmed by a thousand images observed in the visual areas of monkeys [29]. Pattern recognition is a data processing procedure in mammalian brains; proving that perceptions such as vision, pain and hearing are encoded in order to bring meaning to the external world. In order to understand this mechanism and derive a model to present this system there are two ways. The first is a top down hierarchy while the other is the bottom up model used to represent the system. The top down methodology selects a suitable model to fit the data, which requires verification, simulation and validation steps, and is a fast approach. The Bottom up methodology requires more tedious work and a more comprehensive study to work with the data. The advantages of the bottom-up modelling methodology (practiced here) are mainly that (i) can be used for many different types of data, and (ii) the collected data can be recorded from many different areas, which can display a functionally specific region of the brain.

\subsection{For Future Application}

A mathematical function, which is a result of the analysis of a waveforms "data". These waveforms store important information that can be used to describe a network simulation. The response characteristic of a network model was similar "functionally" to the neuronal network (mentioned above). It is therefore possible to devise an electronic circuit from the described network for simulation, which can be utilized as an emulator. This similar hardware approach was also explored for simulation of a neuronal network [30].

The motivation of mathematical modelling studies has been explored in:

(i) The Gaussian function related to modelling studies has already been explored; an estimation theory model in sound localization [31], for the decoding of sound positioning [32], has been better explained for a rat's somatosensory cortex [33].

(ii) The importance of mathematical modelling studies are also emphasized in other applications: (a) An experiment on the behavior of animals in the investigation of dopamine responsive cells, [34], (b) The grid cell firing patterns explored; to build a mathematical model to observe the grid cells' behavior [35], and (c) Modelling demand has drastically been increased in natural sciences which many companies are developing mathematical modelling toolboxes for [36].

(iii) It is not unusual to describe complicated system's dynamics with simple mathematical models [37]; such as, The Mandelbrot set is a type of fractal geometry that can be defined as " $Z_{n}+1=\left(Z_{n}\right)^{2}+c$ " [38], or the equation for intelligence is simple as " $\mathrm{F}=\mathrm{T} \nabla \mathrm{S}_{\tau}$ " [39]. 
The result of the ILD pattern analysis concludes that a meaningful mathematical model can be developed, and this model is as simple as a $1^{\text {st }}$ order Gaussian function; it can be used to represent all varying ILD patterns by varying only the three coefficient values " $a_{1}$ ", " $b_{1}$ " and " $c_{1}$ " of a $1^{\text {st }}$ order Gaussian function.

\subsection{What Is the Reason Behind the Gaussian Function?}

If the brain is considered to be a system then a simple building blocks are individual neurons with their stochastic behaviors [3]. The different number and types of individual neurons can be grouped to construct neuronal networks, and groups of these neural networks outline the functionally specific regions, and the intrinsic structure of many functionally specific regions may put together for an effective information processor pathway. The size, complexity, morphology even the capability these building block structures can be varied; yet the function ought to be an energy-efficient.

Our brains operate with a functional efficiency [40]; a single action potential generation is based on the inside " $\mathrm{V}_{\text {in }}$ " and outside " $\mathrm{V}_{\text {out }}$ " of the potential differences in a nerve cell's membrane and can be explained with the equilibrium of ions ( $\left.\mathrm{I}_{\text {inside }}\right)$ with $\left(\mathrm{I}_{\text {outside }}\right)$ the concentration found by [41]. More than a century old Nernst's-equation which, defines the voltage differences " $\mathrm{V}_{\text {differences" }}$ of a cells membrane potential with a logarithmic function of the ions ratio across channels [42]. This equation (4) can also be rewritten as an exponential function (i.e. in the Eq.17.1 of [43]) with this equation being similar to a $1^{\text {st }}$ order Gaussian function. The Nernst Equation can be reorganized with the parameters of " $F$ " is Faraday's constant, " $R$ " is the gas constant, "T" is absolute temperature $\left({ }^{\circ} \mathrm{K}\right)$, and " $\mathrm{Z}$ " is the valence of the ion, where $\mathrm{Z} . \xi \approx 25 \mathrm{mV}$ at the room temperature.

$$
V_{\text {in }}-V_{\text {out }}=V_{\text {differences }}=\xi \ln \frac{\mathrm{I}_{\text {out }}}{\mathrm{I}_{\text {in }}}, \therefore \frac{\mathrm{I}_{\text {out }}}{\mathrm{I}_{\text {in }}}=e^{\left\{\frac{V_{\text {differences }}}{\xi}\right\}} \text { where, } \xi=\frac{\mathrm{R} \text {.T }}{\text { Z.F }}
$$

The Nernst equation becomes $\mathrm{V}_{\mathrm{i}, 0} \approx(25 \mathrm{mV} / \mathrm{Z}) \times \ln \left(\mathrm{I}_{\text {out }} / \mathrm{I}_{\text {in }}\right)$ at room temperature, and rewritten as $\mathrm{I}_{\text {out }} / \mathrm{I}_{\text {in }} \approx$ $\mathrm{e}^{[\mathrm{V} /(25 \mathrm{mV} / \mathrm{Z})]},[40]$ or a form of a $1^{\text {st }}$ order Gaussian function as in $\mathrm{I}_{\mathrm{o}, \mathrm{i}}=\mathrm{a} \cdot \mathrm{e}^{[\mathrm{b} \cdot V / \mathrm{c}]},[43]$. This equation is not different than the result of the linear relation of the ILD patterns; where, the function-parameters (" $a$ ", " $b$ ", and ' $c$ ") can be read as the physiological variation of the ILD patterns that are generated by the neural circuitry of the IC, (2). Using an over a century old Nernst's equation emphasizes the advantage of a mathematical model over a developed algorithm of the neural network model.

\subsection{The Reconstruction of ILD Patterns}

All ILD patterns can be mathematically reconstructed by the variation of the parameters of $1^{\text {st }}$ order Gaussian function. This effective deduction was based on the reasons:

\subsubsection{Physiological aspects}

(i) ILD data were from the functionally specific region "ICc" of the rat's brain; it refers the neural network can be classified data and can be expressed as a group of patterns,

(ii) Due to the data collection procedure, the stimuli were systematically varied during the sound localization experiment, that signifies the response characteristic of the system the results also showed systematic variations,

(iii) The nerve cell axon is either excitatory or inhibitory that refers the formation of ILD patterns are based on the interactions between excitation and inhibition in ICc,

(iv) The brain's energy efficiency mechanisms can be related to the reasons of the nerve cell's stochastic behavior, or a Homeostatic regulation in the brain, or a Gaussian waveforms "i.e. ILD patterns" in this study, and

(v) The Gaussian functions have already been used in the technological system/ device designs of 
mobile technologies where the energy efficiency is the most important subject matter (such as; to minimizing the energy consumption for mobile devices [44], designing the energy-efficient wireless sensor networks architecture [45] and image transmission [46]).

\subsubsection{Curve fitting for the mathematical modelling}

(i) Seven types of ILD patterns are used as a master-template based on classified ILD data,

(ii) ILD patterns visually inspected if there was a resemblance to "known" a non-discreet function; the result was the normal distribution with varying two parameters of $p d f$. This result was the initiation of the Gaussian type selection for a suitable curve fitting,

(iii) A Gaussian function used as a curve fit for a single ILD pattern was not sufficient to bring up a mathematical model. Therefore, a seven set of coefficients from seven curve fitted functions that all were examined (for the linearity, etc.) for all seven ILD patterns. The linear variation of the coefficients (used in an order entry) that suggests the parameterized $1^{\text {st }}$ order Gaussian function can only be used as a mathematical model.

\section{Conclusion}

Parameterized $1^{\text {st }}$ order Gaussian equation can be used as a mathematical model for describing ILD patterns. In other word, the ILD patterns can be reconstructed by the systematically varying parameters "parameterized" of the $1^{\text {st }}$ order Gaussian function. This conclusion was derived from two reasons: $(a)$ Physiological aspects, and (b) Curve fitting. These are detailed in the Subsection 4.5.

The information (i.e. ILD cues in sound localization) perceived from the sensory system to be encoded for the biologically "plausible" neural networks, as if mathematically discrete function. This encoded information (within the temporal and place principles) is conveyed through the functional regions of the auditory pathway in (ICc) the brain, wherein constructed waveforms "patterns". The collection of these patterns can be functionally described as a mathematical model and this practical procedure proposed here with the intention to help the readers for their future applications in machine learning.

\section{Appendix}

Seven pdf distributions were chosen among 432 pdf plots, and only 252 windows (with varying $\mu$ and $\sigma$ ) can be observed in the G-Drive "https://drive.google.com/file/d/0B0duvNF-bLB8ME1CeWZIbkY1UTg". The figures (Fig. 1, Fig. 2 and Fig. 3) can also be seen clearly in the G-Drive, the link can be accessed through the "https://drive.google.com/file/d/1t0-30s5HmAb64AeZsnrC6FiSeAUGy9gS".

\section{Acknowledgment}

The author has received ILD data set for normalization and classification studies from his PhD supervisor whom had been the co-author of both manuscripts in [5], [47].

\section{References}

[1] Johnson, H. R., et al. (2016). A machine learning approach for using the postmortem skin microbiome to estimate the postmortem interval. PLOS ONE, 11(12), e0167370.

[2] Nasir, M., Baucom, B., Narayanan, S. S., \& Georgiou, P. (2016). Complexity in prosody: A nonlinear dynamical systems approach for dyadic conversations; behavior and outcomes in couples therapy. Interspeech, 893-897.

[3] Vries, B., \& Dittberner, A. (2013). Is hearing-aid signal processing ready for machine learning? Proceedings of the International Symposium on Auditory and Audiological Research (vol. 4, pp. 121-132).

[4] Aitkin, L. (1990). The Auditory Cortex: Structural and Functional Bases of Auditory Perception. $1^{\text {st }}$ ed. 
London, UK: Chapman \& Hal.

[5] Uragun, B., \& Rajan, R. (2013). The discrimination of interaural level difference sensitivity functions: Development of a taxonomic data template for modelling. (in Eng). BMC Neuroscience, 14(1), 114.

[6] Wise, L. Z., \& Irvine, D. R. (1985). Topographic organization of interaural intensity difference sensitivity in deep layers of cat superior colliculus. Journal of Neurophysiology, 54(2).

[7] Park, T. J., Monsivais, P., \& Pollak, G. D. (1997). Processing of interaural intensity differences in the LSO: Role of interaural threshold differences. Journal of Neurophysiology, 77(6), 2863-2878.

[8] Pollak, G. D., Burger, R. M., Park, T. J., Klug, A., \& Bauer, E. E. (2002). Roles of inhibition for transforming binaural properties in the brainstem auditory system. Hearing Research, 168(1-2), 60-78.

[9] Pena, J. L., \& Konishi, M. (2004). Robustness of multiplicative processes in auditory spatial tuning. The Journal of Neuroscience, 24(40), 8907.

[10] Groh, J. M., Kelly, K. A., \& Underhill, A. M. (2003). A monotonic code for sound azimuth in primate inferior colliculus. Journal of Cognitive Neuroscience, 15(8), 1217-1231.

[11] Patterson, R. D. (1974). Auditory filter shape. The Journal of the Acoustical Society of America, 55(4).

[12] Lin, L., Ambikairajah, E., \& Holmes, W. (2001). Log-magnitude modelling of auditory tuning curves. IEEE International Conference on Acoustics, Speech, and Signal Processing. Salt Lake City, UT, USA.

[13] Spitzer, P., Zierhofer, C., \& Hochmair, E. (2006). Algorithm for multi-curve-fitting with shared parameters and a possible application in evoked compound action potential measurements. Biomedical Engineering, 5(13).

[14] Ponton, C. W., Eggermont, J. J., Coupland, S. G., \& Winkelaar, R. (1993). The relation between head size and auditory brain-stem response interpeak latency maturation. The Journal of the Acoustical Society of America, 94(4).

[15] Vivion, M. C. (1975). Recovery of middle components of human auditory averaged electroencephalic responses. The Journal of the Acoustical Society of America, 57(S1), S62.

[16] Finneran, J. J., \& Hastings, M. C. (2000). A mathematical analysis of the peripheral auditory system mechanics in the goldfish (Carassius auratus). The Journal of the Acoustical Society of America, 108(3), 1308-21.

[17] Eggermont, J. J. (1989). Coding of free field intensity in the auditory midbrain of the leopard frog. I. Results for tonal stimuli. Hearing Research, 40(1-2), 147-165.

[18] Uragun, B. (2015). An automatic recognition for the Auditory Brainstem Response waveform. Proceedings of $14^{\text {th }}$ International Conference on Machine Learning and Applications and Workshops (pp. 423-8).

[19] Uragun, B. (2017). Using ANN back-propagation technique to represent the group of ILD patterns. Proceedings of $6^{\text {th }}$ International Conference on Informatics, Electronics \& Vision (ICIEV) \& $7^{\text {th }}$ International Symposium in Computational Medical and Health Technology (ISCMHT). University of Hyogo, Himeji, Japan, IEEE.

[20] Whittle, P. (1958). On the smoothing of probability density functions. Journal of the Royal Statistical Society. Series B (Methodological), 20(2), 334-343.

[21] Lukacs, E. (1942). A characterization of the normal distribution. Ann. Math. Statist., 91-93.

[22] Tuckwell, H. C. (1989). Stochastic processes in the neurosciences (CBMS-NSF regional conference series in applied mathematics). Philadelphia, PA, USA: Society for Industrial and Applied Mathematics.

[23] Uragun, B. (2011). Nano aerial vehicles as flapping wing robots. Fifth International Workshop on Robotics for risky interventions and Environmental Surveillance-Maintenance. Leuven, Belgium.

[24] Muijres, F., \& Dickinson, M. (2014). Bird flight: Fly with a little flap from your friends. Nature, 505(7483). 
[25] Adam, J. A. (2006). Mathematics in Nature: Modeling Patterns in the Natural World. Princeton University press, NJ, USA.

[26] Parise, C. V., Knorre, K., \& Ernst, M. O. (2014). Natural auditory scene statistics shapes human spatial hearing. Proceedings of the National Academy of Sciences, 111(16), 6104-6108.

[27] Afraimovich, V., Gong, X., \& Rabinovich, M. (2015). Sequential memory: Binding dynamics. Chaos, 25(10).

[28] Johnson, N. F., et al. (2013). Simple mathematical law benchmarks human confrontations. Scientific Reports, 3.

[29] Majaj, N. J., Hong, H., Solomon, E. A., \& DiCarlo, J. J. (2015). Simple learned weighted sums of inferior temporal neuronal firing rates accurately predict human core object recognition performance. Neuroscience, 35(39).

[30] Lewis, E. R. (1968). Using electronic circuits to model simple neuroelectric interactions. IEEE, 56(6).

[31] Divenyi, P. L., \& Zakarauskas, P. (1992). The effect of bandwidth on auditory localization: An estimation theory model. Auditory Physiology and Perception 9th International Symposium on Hearing. Carcens, France.

[32] Mlynarski, W. (2014). Efficient coding of spectrotemporal binaural sounds leads to emergence of the auditory space representation. Frontiers in computational neuroscience, 8, 1-13.

[33] Maass, W., Natschlaeger, T., \& Markram, H. (2004). Computational models for generic cortical microcircuits. Computational Neuroscience: Comprehensive Approach. (Chapman \& Hall/ CRC) London, UK.

[34] Gurney, K. N., Humphries, M. D., \& Redgrave, P. (2015). A new framework for cortico-striatal plasticity: Behavioural theory meets in vitro data at the reinforcement-action interface. PLoS Biology, 13(1).

[35] Krupic, J., Bauza, M., Burton, S., Barry, C., \& O’Keefe, J. (2015). Grid cell symmetry is shaped by environmental geometry. Nature, 518(7538), 232-235.

[36] Buckingham, S. D. (2007). To build a better model. Nature Methods, 4(4), 367-374.

[37] May, R. M. (1976). Simple mathematical models with very complicated dynamics. Nature, 261(5560), 459-467.

[38] Mandelbrot, B. (1982). The Fractal Geometry of Nature. New York, NY, USA.

[39] Wissner-Gross, A. D., \& Freer, C. E. (2013). Causal entropic forces. Physical Review Letters, 110(16), 168702.

[40] Iaizzo, P. A. (2013). Introduction to neurophysiology. Neural Engineering. Boston, MA, USA: Springer.

[41] Nernst, W. (1889). On the kinetics of bodies in solution. Philosophical Magazine Series, 5(28), 171.

[42] Chappell, M., \& Payne, S. (2016). The action potential. in Physiology for Engineers, 13. Rome, Italy: Springer.

[43] Phillips, R. (2012). Biological electricity and the hodgkin-huxley model. Physical Biology of the Cell. New York, NY, USA: Garland Science.

[44] Ma, S. (2014). Improving the performance and energy efficiency for mobile cloud computing. MSc, Computer Science, San José State, San José, CA, USA, 418.

[45] Peckens, C. A., Lynch, J. P., \& Heo, G. (2015). Resource-efficient wireless sensor network architecture based on bio-mimicry of the mammalian auditory system. Journal of Intelligent Material Systems and Structures, 26(1), 79-100.

[46] Aziz, S. M., \& Pham, D. M. (2013). Energy efficient image transmission in wireless multimedia sensor networks. IEEE communications letters, 17(6), 1084-1087.

[47] Uragun, B., \& Rajan, R. (2011). Developing an appropriate data normalization method. Proceedings of Tenth International Conference on Machine Learning and Applications and Workshops. Honolulu, HI, US, 
vol. 2: IEEE.

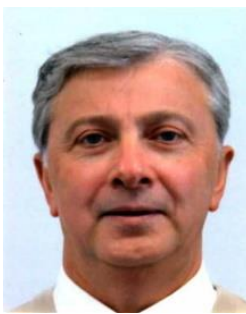

Balemir Uragun has received a BSc in electronics engineering from Istanbul Technical University, Turkey and the MSc in electrical engineering from Tufts University, MA, USA. He has been working for several defense and research projects in the industries where the positions held from a senior research-fellow to a project-manager. His field of interest is in the mathematical modelling area. During his professional works and his PhD study, he published several papers on the topic of Energy Efficiency, Noise Reduction, ANN, Data classification, and data normalization, Pattern Recognition, and Machine Learning. 